\title{
Image-based characterization of powder flow to predict the success of pharmaceutical minitablet manufacturing
}

\section{Blanco, David}

2020-05-15

Blanco , D , Antikainen , O , Räikkönen , H, Pei Ting Mah , Healy , A M , Juppo , A M \& Yliruusi , J 2020 , ' Image-based characterization of powder flow to predict the success of pharmaceutical minitablet manufacturing ' , International Journal of Pharmaceutics , vol. 581 , 119280 . https://doi.org/10.1016/j.jpharm.2020.119280

http://hdl.handle.net/10138/324439

https://doi.org/10.1016/j.jpharm.2020.119280

unspecified

acceptedVersion

Downloaded from Helda, University of Helsinki institutional repository.

This is an electronic reprint of the original article.

This reprint may differ from the original in pagination and typographic detail.

Please cite the original version. 


\title{
Image-based Characterization of Powder Flow to Predict the Success of Pharmaceutical Minitablet Manufacturing
}

\author{
David Blanco a,*, Osmo Antikainen a, Heikki Räikkönen a , Pei Ting Mah b, Anne Marie Healy b,c, Anne Mari Juppo a, Jouko Yliruusi a. \\ ${ }^{a}$ Division of Pharmaceutical Chemistry and Technology, Faculty of Pharmacy, University of Helsinki, P.O. Box 56, (Viikinkaari 5E), FIN-00014, Finland \\ ${ }^{\mathrm{b}}$ School of Pharmacy and Pharmaceutical Sciences, Panoz Institute, Trinity College Dublin, Dublin 2, Ireland. \\ ${ }^{\mathrm{c}}$ SSPC, Synthesis and Solid State Pharmaceutical Centre, Ireland. \\ * Corresponding author. E-mail address: david.blanco(@)helsinki.fi
}

5

Keywords: Powder flow, Cohesive powders, Tableting, Microcrystalline cellulose, Image analysis, Small scale.

\section{ABSTRACT}

Powder flowability plays an important role in die filling during tablet manufacturing. The present study introduces a novel small-scale measuring technique for powder flow. Based on image analysis, the flow was defined depending on the variation of luminous intensity and the movement of powder inside the measurement cuvette. Using quantities around $100 \mathrm{mg}$ it was possible to characterize a wide range of common pharmaceutical powders, especially in distinguishing subtle differences in flow caused by minor changes in samples characteristics. The method was compared with powder rheometry, which is widely used in the pharmaceutical literature, and showed a significant improvement in predicting the success of pharmaceutical minitablet manufacture $(\mathrm{d}=5 \mathrm{~mm})$. Tablet weight variation $(\mathrm{RSD})$ was defined as the most efficient way to assess relevant powder flow behaviour in tablet production when using the novel device. The proposed method was distinguished from others by its ability to classify different grades of microcrystalline cellulose in the die-filling process. Subsequently, eight common pharmaceutical powders, both excipients and APIs, were properly ranked as a function of flowability based on their physical properties. The method showed a high repeatability, with a relative standard deviation not more than $10 \%$.

\section{INTRODUCTION}

Powder flow has been studied for decades in the fields of metallurgy, the food industry and the pharmaceutical industry. The importance of powder flow and the consequences of poor flow are well known $(1,3,4,7,8)$. Still, poor powder flow is a frequently encountered challenge in drug product manufacturing. Currently the most widely used techniques to measure powder flow involve using different kinds of funnels or orifices through which the powder flows, while an important aspect - powder flow on the unit dose volume level and below - has received less attention $(1,3,16)$. The funnel and orifice-based systems are easy to use, but their practical significance is limited $(2,3,13)$. Especially in pharmaceutical technology, bulk and tapped densities and various derived calculated parameters have been used as indirect measures of powder flow (5). However, those methods cannot provide detailed information on flowability, since the physical characteristics of the particles affect more than interparticular forces. There is a clear gap in knowledge of the characteristics of the flow of small volumes of powder in confined spaces, where particle-level behavior dominates over bulk behavior $(2,19)$.

In the pharmaceutical industry the impact of this problem is extensive; proper powder flow is important in the manufacturing, transportation and accurate dispensing of powders for the majority of products (3). For example, in powder compression processes, flowability is affected by the physical properties of the powder, the state of the powder (e.g. free flowing, tap, etc.), the type of loading (e.g. air flow, forced feeding, gravity, etc.) and the environmental conditions. Tablet weight variation must be within a specified limit, and it is essential to understand the powder flow properties in order to avoid tablets of unacceptable quality. The economic impact of this problem is equally important. The present study aims to address this largely neglected, but fundamentally important research topic. 
The most sensitive powder flow meters today seem to be shear cells and powder rheometers $(3,6,20)$. However, these provide indirect methods of flow, and single determinations require large sample sizes (typically over 30 grams). It has also been recognized that the results between methods are, at times, contradictory, or do not correlate with each other (10), i.e. the method of analysis may well show unreliable results on powder flow properties, depending on the method of measurement more than the significance to the process. No single technique, nor mathematical model, is suitable for the full characterization of a powder $(3,4,11)$; different methods must be employed to completely understand the flow properties, which can never be expressed as a single value (18). Consequently, current devices can be used only for comparative testing, rather than process design (14). Furthermore, the smaller the powder particle size, the poorer is the flowability $(11,12)$. It has been said, however, that there is no suitable method to determine the flowability of very poorly flowing powders (21-24), where the interparticulate cohesive forces dominate over gravitational forces. In addition to traditional tablet and capsule manufacturing, other applications, in which a unit dose is created and powder flow is a concern, are of relevance in the pharmaceutical sector. This is the case, for example, in dry powder inhaler formulations and in the case of the electrostatic deposition of active drug onto tablets. These and other novel processes require new tools to characterize the effect of powder flow during the creation of the dosage form $(3,39-41,43)$.

In recent years, different analytical techniques based on direct high-speed imaging and particle image velocimetry (PIV) have been used to measure solid particle flow $(17,36-38,44)$. Image-analysis technique proved to be a simple tool for the non-invasive study of flow patterns and agglomeration behaviour of fluidized solids $(35,42)$. In contrast to systems designed for this propose which based their analysis on the fluidization process -suspending the powder particles with a fluid-, the novel device mobilizes the powder through an accelerated linear motion, barely subject to the fluidization process. The main aim of the present study was to develop a novel method to study the flow of small sample amounts of pharmaceutical powders in a confined space, with an emphasis on particle-level understanding of the flow in minitablet manufacturing. This might be useful to predict the challenges appearing when entering to large-scale production.

55

\section{MATERIALS AND METHODS}

\subsection{Materials}

Eight common pharmaceutical powders, both excipients and active pharmaceutical ingredients (API's), were selected for this study based on their different individual particle properties and bulk properties (i.e. particle size and shape, density or surface area), in other words, different flow profiles. Lactose monohydrate (Pharmatose 80M sieve lactose and Pharmatose 200M milled lactose, Ph.Eur grade, DmV-Fonterra Excipients, Veghel, Netherlands), standard microcrystalline cellulose (MCC; Vivapur ${ }^{\circledR}$ 101, Ph.Eur grade, JRS Pharma, Rosenberg, Germany; Avicel ${ }^{\circledR}$ PH-102, Ph.Eur grade, FMC BioPolymer, Ireland; Avicel® PH-200, Ph.Eur grade, JRS Pharma, Rosenberg, Germany), national starch (Chemical GmbH, Espoo, Finland), paracetamol (Ph.Eur grade, ORIOLA, Espoo, Finland) and caffeine (Ph.Eur grade, ORIOLA, Espoo, Finland), were used to investigate the differences between flow characterization techniques. No lubricant was added to the samples. Special emphasis was placed on substances with poor flow properties. All the selected materials are electrical insulators, therefore the relative humidity (RH) was critical to their flow behaviour. Polylactic acid (PLA) bioplastic was chosen as 3D printing feedstock material.

\subsection{Sample preparation and acclimatization}

Prior to subject to any analysis, all powders were manually sieved through a $1 \mathrm{~mm}$ standard sieve to remove possible lumps derived from consolidation 
drying temperature specifications in the Ph.Eur. Every 15 minutes the loss of mass was monitored by obtaining the weight until no significant change was detected between three consecutives measurements, and the sample was then considered to be completely dry.

A RH of $50.0 \pm 4.1 \%$ was used in all analytical procedures to simulate pharmaceutical manufacturing processes. After drying, samples were stored for at least 5 days in a desiccator containing a saturated solution of $\mathrm{Ca}\left(\mathrm{NO}_{3}\right)_{2} \cdot 4 \mathrm{H}_{2} \mathrm{O}\left(50.5 \% \mathrm{RH}\right.$ at $\left.25^{\circ} \mathrm{C}\right)$. Humidity conditions were registered for every measurement (F007TH Wireless Thermo-Hygrometer Ambient Weather; Chandler, AZ, USA).

\subsection{Powder Physical Properties: Size, morphology and true density}

Particle size distribution (PSD) and true density were measured in triplicate and are reported in Table 1. PSD along with specific surface area (SSA) were determined using the dry dispersion technique with a laser diffractometer Malvern Mastersizer 3000 instrument (Malvern Instruments Ltd.,

80 Worcestershire, U.K.). Dry powder samples were analyzed using the Aero S dry powder disperser, equipped with a micro volume sample tray (2-10g), applying 3 bar dispersive air pressure and 50\% vibration feed rate. MS3000 software (v3.5) generated particle size distribution data. True density of dried powder samples was measured using a Micromeritics 1305 Helium Pycnometer (Norcross, USA).

Particle morphology was examined using a scanning electron microscope (Quanta FEG250 SEM, ThermoFisher, Oregon, USA), equipped with a large field low vacuum SED (LFD) detector. The samples were sputtered with gold prior to the microscopic examination. SEM micrographs are reported in Fig.

\subsubsection{Water Sorption Isotherms}

Surface water activity, or vapor pressure of water in samples, was determined using an AquaLab Model Series 3TE (Decagon Devices, Inc., Washington, USA). Loss on drying (LOD) was measured thermogravimetrically using a Moisture Analyzer (MA100, Sartorius AG, Goettingen, Germany) and the temperature specifications detailed in the pharmacopoeia. All measurements were undertaken in triplicate prior to and after stored for at least 5 days in a desiccator containing a saturated solution of $\mathrm{Ca}\left(\mathrm{NO}_{3}\right)_{2} .4 \mathrm{H}_{2} \mathrm{O}\left(50.5 \% \mathrm{RH}\right.$ at $\left.25^{\circ} \mathrm{C}\right)$.

\subsection{Powder flow properties}

Considering the variety of methods for characterizing powder flow, those that have appeared most frequently in the pharmaceutical literature were selected to characterize the materials and provide a powder flow reference for the novel method introduced here. Only certain methods can describe the die-cavity filling in compression processes, -i.e. dynamic flow-. Therefore, some commonly-used methods were directly excluded from the study as nonpotential descriptors for powder flow; 1) From Ph.Eur, angle of repose (AOR) cannot be used to assess how materials flow into a bin or tablet press (31) and is only applicable for powders with low and intermediate cohesion since there is not a well-defined angle of repose for more cohesive powders; 2) Shear cell testers, such as FT4 static analysis or Jenike shear tester (32), are useful to predict the behaviour of powder in steady-state flow, but the link between these test and dynamic flow is not clear (33). Conversely, flow function (FF) and cohesion, which are commonly used parameters to rank flowability were included in the analysis.

From the Ph.Eur (5), Hausner Ratio (HR), Carr’s Compressibility Index (CI), and flow rate through an orifice were determined in triplicate (n=3). In addition, FT4 powder rheometer (Freeman Technology, Worcestershire, UK) measurements were undertaken due to its wide use in the pharmaceutical industry to characterize powder flow properties. Especially, the tablet weight variation (Ph.Eur) -as an indicator of the influence of powder flow properties 
on the manufacturing processes- was used to examine the ability of multiple flow parameters to predict/correlate with the success of tablet manufacturing. For that specific purposes only directly compressed materials were analyzed; these are the three different grades of microcrystalline cellulose.

\subsubsection{Pharmacopoeial flow-testing methods}

HR and CI parameters were derived from tapping studies using the procedure "Measurement in a graduated cylinder" and Erweka S VM equipment (Erweka Apparatebau GmbH, Germany). The flow through an orifice was determined measuring the flow rate for 50 grams of powder in a pharmacopoeia (Ph.Eur 10th Ed. 2.9.36) standardized funnel (d=9mm). All the measurements were done in triplicate.

\subsubsection{FT4 Powder Rheometer/Shear cell}

Both dynamic and static powder flow properties were characterized using a FT4 powder rheometer (Freeman Technology, Worcestershire, UK). The device was calibrated prior to analysis in terms of height, force and torque. All the analysis protocols were integrated with conditioning and test cycles (26). For dynamic analysis the powder samples were filled in a $25 \mathrm{~mm}$ x $25 \mathrm{~mL}$ splitting cylindrical vessel and conditioning was performed using a precision impeller blade with a helix angle of $-5^{\circ}$ and $23.5 \mathrm{~mm}$ in diameter. The dynamic flow test and the variable flow rate test method were performed using a blade tip speed of $100 \mathrm{~mm} / \mathrm{s}$ for the former and 100, 70, 40 and $10 \mathrm{~mm} / \mathrm{s}$ for the latter (26). The conditioned bulk density (CBD), specific energy (SE), stability index (SI), flow rate index (FRI) and basic flowability energy (BFE) data were generated from the dynamic powder flow test (6).

The static analysis was performed using a shear cell head accessory (6). The cohesion (kPa), the flow function (FF) and wall friction angles (WFA) or angles of internal friction (AIF) were determined using the rotational $1 \mathrm{ml}$ shear cell module where the powder samples were conditioned using conditioning wire and pre-compressed using $9 \mathrm{kPa}$ consolidation stress by a vented piston. The shear stress (kPa) values were measured at $9,7,6,5,4$ and $3 \mathrm{kPa}$ applied normal stress. The standard wall friction test program was used to perform the analysis using a wall friction head equipped with a stainless steel disc with $0.05 \mathrm{~mm}$ surface roughness $(6,28)$. Only cohesion and FF parameters were included in the analysis.

125

\subsubsection{Novel In-house flow testing device}

- Structure and principle of measurement

The device comprises a sample cuvette mounted on a stepper motor, which is controlled with a custom-made software. It was designed and assembled using 3D modelling software (FreeCAD v.0.35, and Ultimaker Cura v3.4.1) and a fused filament fabrication 3D printer (Ultimaker 2+, Ultimaker B.V, Utrecht, Netherlands). In order to integrate all the components of the system, a robust central module was designed by means of 3D printing (see Fig. 1). This module is connected to the mobile platform of the step motor structure using 4 vibration-free steel shafts. It includes, from bottom to top: 1. The light source (LED-lamp, Biltema, Helsinki, Finland). 2. Above the lamp, the sample cuvette is enclosed in a compartment sealed to external light. As the measurement cuvette is closed, there is not contamination or environmental exposure. The base, where the cuvette is located, has an opening that allows direct illumination of powder samples. 3. From above, powder flow inside the cuvette was monitored by means of real-time digital imaging. 


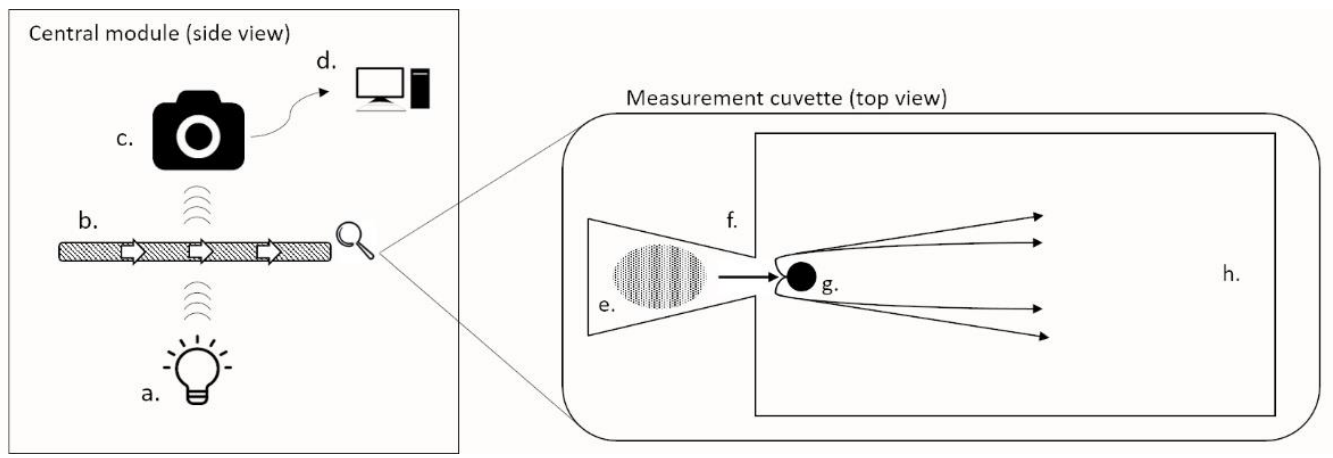

Fig. 1

Scheme of the powder flow measuring device testing set-up, including a detailed diagram of the measurement cuvette (not to scale) and the powder flow inside. a. Light source.

b. Sample Cuvette. c. Camera module. d. Computer, data acquisition. e. Loading chamber, powder sample inside. f. Orifice. g. Disturbance pivot. h. Analytical chamber.

* White arrows represent the direction of movement of the device. Black arrows present the powder flow inside the measurement cuvette.

The cuvette consists of two horizontally positioned chambers separated by an orifice (d=6mm), shown in detail in Fig. 1. The powder sample, with a standard fixed volume of $0.2 \mathrm{~cm}^{3}$, was placed in the left chamber -loading chamber-. Then, the cuvette was subjected to a specific acceleration profile, consisting of a slow acceleration and rapid deceleration when moving to the right (see more in the next section "Motion profile"). Consequently, based on

145 Newtonian mechanics, the powder moved to the opposite chamber -analytical chamber- through the orifice. In the analytical chamber, an aluminum pivot was placed behind the orifice, generating a disturbing point to the powder flow. This, resulted in the creation of unique patterns for each sample analyzed when the powder had completely transferred to the analytical chamber.

\section{- Standardization process}

In order to reduce human intervention as much as possible, a simple feeding accessory for sample weighing and loading into the cuvette was designed by means of 3D-printing. This resulted in a standard test that produced a low stress packing state and highly reproducible sample deposition between measurements. Consisting of a small aluminium funnel and a manual closure of the lower aperture ( $4 \mathrm{~mm}$ in diameter), the samples were weighted inside (aperture closed) and then placed in the measurement cuvette. The external structure of PLA, whose shape perfectly matched the loading chamber (Fig. 1), allowed a reproducible loading of the powder sample when the funnel's aperture was opened. The systematic optical evaluation confirmed that powder's sample shape and position were similar between the measurements.

Static charge is one of the main limitations when handling small particle size materials $(8,12)$. However, it was observed that using earth connections, aluminum components, and washing the cuvette and the feeding accessory with water and dried pressured air between measurements, the flow behaviour was not significantly affected by the electrical charges of the system. High repeatability on the starting point of movement was ensured by pre-establiched conditioning of the device. This was based on the systematic calibration of the starting point at the beginning of each analysis. Using a mechanical system (switch), the rotation of the engine was automatically reset to exactly the same position every measurement.

The selection of the analytical sample size in the cuvette was determined volumetrically. Different materials were optically evaluated to estimate the optimal amount of sample needed for the most efficient determination of powder flow properties. This is defined as the sample volume needed to adequately produce the powder flow pattern with a minimum powder residue in the loading chamber. The optimal volume was $0.2 \mathrm{~cm}^{3}$, and subsequent measurements confirmed this sample volume as ideal. In addition, this also validated that the surface area of the analytical chamber in the cuvette was appropriate for the dispersion analysis of all materials tested in this study. Since the load on the cuvette was determined by weighing, bulk density was 
used to calculate the ideal sample size in milligrams, which resulted within a range from $57 \mathrm{mg}$ (Paracetamol; smallest Dv50=20.7 $\mu \mathrm{m})$ to $117 \mathrm{mg}$ (Lactose 80M; largest Dv50=212.7 $\mu \mathrm{m}$ ). The repeatability of the novel powder flow method was determined by calculating the standard deviation and RSD from 20 flow measurements of Avicel® PH-102 (StDev=2.59 and RSD=9.02). A constant volume of $0.2 \mathrm{~cm}^{3}$ or sample size of $77 \pm 0.5 \mathrm{mg}$ was used.

\section{- Motion profile}

Two high-accuracy laser displacement sensors (Keyence LK-H087, Keyence Corporation of America, Itasca, Illinois, USA) were used to measure the displacement of the sample cuvette along the device, corresponding to a total distance of $35 \mathrm{~cm}$. The lasers were connected to a controller (KeyenceG5001P, Keyence Corporation of America, Itasca, Illinois, USA), and time-distance data was recorded using Keyence LK_H3-software (Keyence Corporation of America, Itaska, Illinois, USA). The detection resolution was $1 \mu \mathrm{m}$, sampling rate was $20 \mathrm{kHz}$ and the laser range was set at $36 \mathrm{~mm}$ for higher resolution. To produce the velocity and acceleration data (see Fig. 2), the time-distance data was processed using MatLab (v.2018b, Mathworks Inc., Natick, Massachusetts) and SigmaPlot (Systat Software, San Jose, CA).

For better understanding, the motion profile (Fig. 2) was divided into different phases as follows; a. Resting state - Low acceleration: From the resting state/zero speed, the powder sample in the loading chamber is subjected to positive acceleration to the right. b. Fast deceleration: Subsequently, a higher negative acceleration triggers the migration of the powder sample from the loading chamber to the analytical chamber through the orifice, impacting the disturbance pivot. c. First static state: Zero speed. The sample has been dispersed in the analytical chamber, creating the corresponding unique flowpattern. d. Second static state - Image capturing: In order to homogenize the sample's distribution on the analysis surface, two high-acceleration vibrations are performed.
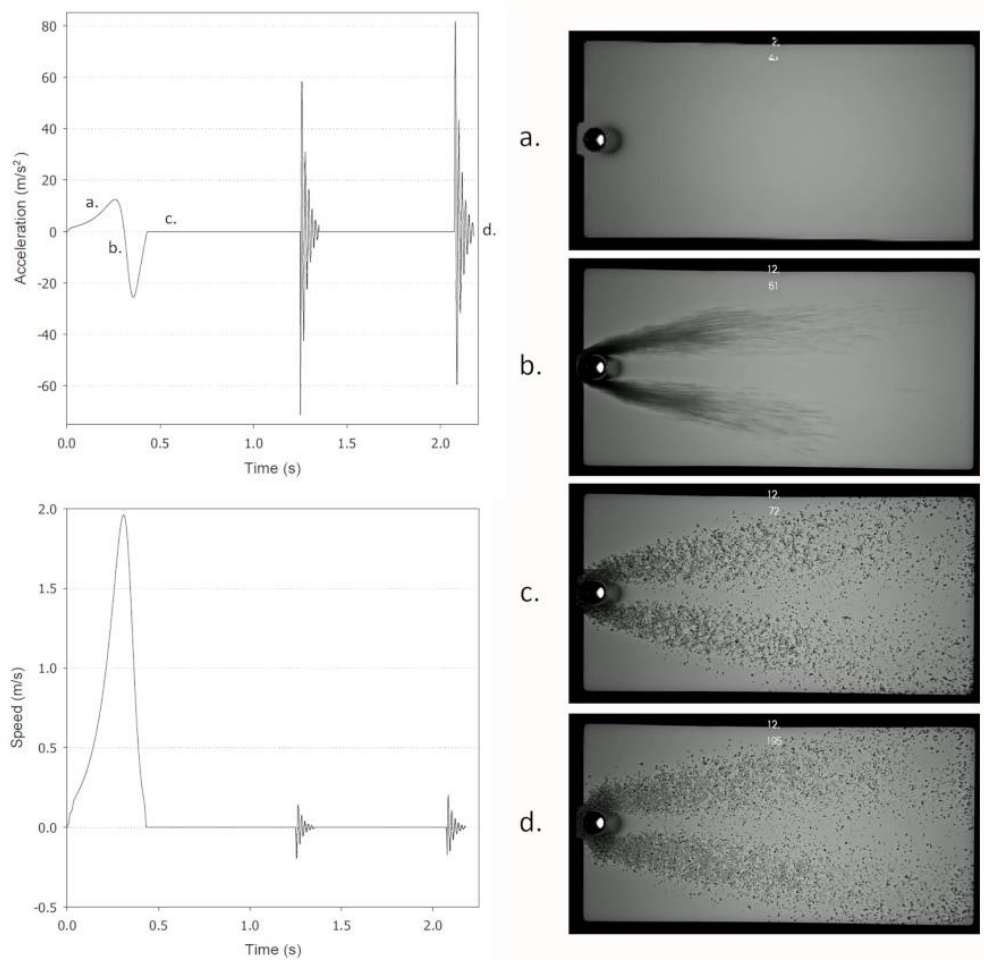

c.

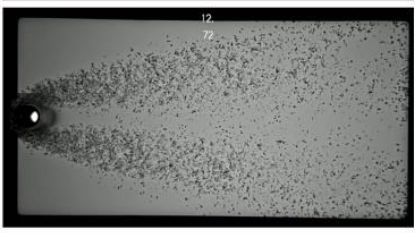

d.

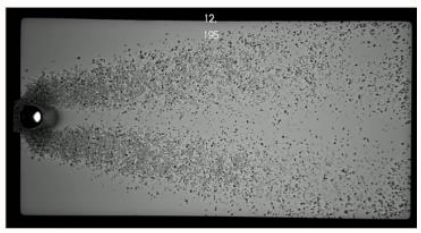

Fig. 2.

Motion profile (acceleration and speed) and the correlation to the different powder-flow stages inside the measurement cuvette.

a. Resting state - Low acceleration. b. Fast deceleration. c. First static stage. d. Second static stage - Image capturing. 
- Data acquisition and processing

Single-board computer Raspberry Pi 3 Model B+ (Raspberry Pi Foundation, United Kingdom) was used in conjunction with the 8-megapixel Raspberry Pi Camera Module v2 (Raspberry Pi Foundation, United Kingdom) and a temperature \& humidity sensor (HYT 221, Innovative Sensor

Technology IST AG, Switzerland). Apart from that, a laptop connected to the step motor was used to monitor the activity by initiating the movement. A user-friendly interface written in Python facilitated the collection and storage of real-time data from each sensor. Data-analysis was performed based on numeric descriptors extracted using MatLab image-analysis. Video data was processed to get a single flow parameter titled Flowability Index (FI\%), which describes the overall flow behaviour. In this study only the first and last images of the video sequence were used for technical reasons.

The principal assumption of the analysis showed that the light intensity captured by the optical sensor in the camera was directly related to the flow of powder sample in the analytical chamber. First, the image of the empty cuvette (before movement started, Fig. 2a.) was analyzed by calculating in grey scale (values from 1 to 255) the background light intensity for each pixel using MatLab Boolean functions. Then this operation was repeated for the last image (end of the movement, Fig. 2d). Both images were compared on their grey-scale values pixel by pixel. This allowed to standardize the possible variations in background light intensity between pixels, caused by the light source or room light distortion. In case the analyzed pixel showed a darker value when compared to the background pixel, it was colored black, assuming powder particles present in the analytical chamber absorb certain light intensity. Otherwise the pixel was considered "empty" and was colored white. The resulting binary image (black and white) was used to obtain the FI\% parameter as a function of the total percentage of analytical chamber's area covered by powder. This on/off pixel analysis method made the technique independent of certain properties of the material that may affect the light intensity -i.e. obscuration-, such as the degree of crystallinity or particle composition, and the different arrangement in layers that the sample may undergo after the dispersion over the analytical surface.

\subsection{Tableting studies}

Pharmaceutical tablets are the most popular dosage form for drug delivery and are normally produced by compressing dry powder inside a die. In the compaction process, three stages can be differentiated; die filling of the powder blend facilitated by gravity or a forced feed system, compaction into a tablet, and ejection of the compressed compact. The importance of powder flow in the die filling, among other factors (33), determines the uniformity of the powder blend and the packing fraction, directly influencing the properties of tablets.

As mentioned above, the lack of any single technique suitable for the full characterization of a powder, and considering the variability of the methods and the number of parameters that affect the flow, it was decided to use inter minitablet weight variation to relate powder flow properties directly to the tablet compression process. For this purpose, and based on bibliographic data, only the studied materials suitable for direct compression -without lubricantwere selected. These were the three grades of MCC, an excipient which is commonly used as filler/binder in direct compression formulations $(9,30)$, owing to its high compressibility and good/acceptable flowability (27). An eccentric tablet press (Type F3, Manesty Machines LTD, Liverpool, England) was used in conjunction with a concave single-punch $\left(\mathrm{d}=5 \mathrm{~mm}, 60\right.$ tablets $\left.\mathrm{min}^{-1}\right)$, the small diameter increased the flow requirements for die filling and provided better characterization of powder flow properties (4). A gravity feeder on the tablet press was selected, which requires better powder flow to attain the same variability in tablet weight RSD in comparison to a force feeder. This study was conducted for three different batches of each MCC grade. Between batches, the equipment was cleaned and the process of loading and filling the feeder was made from the very beginning. The weight of the tablets was analyzed for each batch until relative standard deviation remained stable (RSD<1), considering consistency in the measurement. 


\section{RESULTS AND DISCUSSION}

Relating powder flow properties to behaviour in manufacturing processes is the major reason flowability is measured (3). Flowability results from the combination of material physicochemical properties and the equipment used along the different stages of drug-development (storage, blending, compaction, fluidization, etc.). Therefore, it is very important to use an appropriate strategy to characterize the various aspects of powder, choosing the tests that better approach the flow behaviour $(3,25)$.

\section{Table 1}

Summary for PSD, particle porosity and tapping studies.

\begin{tabular}{|c|c|c|c|c|c|c|c|c|}
\hline & Lactose $80 \mathrm{M}$ & Lactose 200M & Vivapur 101 & Avicel PH102 & Avicel PH200 & Starch & Caffeine & Paracetamol \\
\hline \multicolumn{9}{|l|}{ Size } \\
\hline$d 10(\mu \mathrm{m})$ & $39.8 \pm 2.0$ & $6.2 \pm 0.4$ & $21.5 \pm 0.1$ & $23.4 \pm 0.1$ & $40.8 \pm 0.4$ & $9.7 \pm 0.1$ & $4.7 \pm 0.1$ & $4.0 \pm 0.0$ \\
\hline$d 50(\mu \mathrm{m})$ & $212.7 \pm 2.1$ & $40.1 \pm 0.9$ & $63.2 \pm 0.2$ & $102.7 \pm 0.6$ & $189.3 \pm 1.2$ & $20.8 \pm 0.2$ & $41.0 \pm 0.7$ & $20.7 \pm 0.2$ \\
\hline$d 90(\mu m)$ & $389.7 \pm 2.1$ & $131.3 \pm 2.5$ & $142.3 \pm 0.6$ & $259.3 \pm 0.6$ & $427.7 \pm 2.1$ & $55.9 \pm 0.3$ & $201.3 \pm 8.5$ & $141.7 \pm 10.2$ \\
\hline Span & $1.65 \pm 0.01$ & $3.12 \pm 0.03$ & $1.9 \pm 0.0$ & $2.26 \pm 0.01$ & $2.04 \pm 0.01$ & $13.2 \pm 1.7$ & $4.80 \pm 0.12$ & $6.62 \pm 0.45$ \\
\hline Uniformity & $0.481 \pm 0.005$ & $1.096 \pm 0.026$ & $0.594 \pm 0.002$ & $0.710 \pm 0.004$ & $0.637 \pm 0.005$ & $0.704 \pm 0.003$ & $1.681 \pm 0.091$ & $4.580 \pm 0.628$ \\
\hline Specific Surface Area $(S S A) \mathrm{m}^{2} / \mathrm{kg}$ & $104 \pm 11$ & $431 \pm 25$ & $146 \pm 5$ & $112 \pm 1$ & $65 \pm 1$ & $609 \pm 43$ & $520 \pm 7$ & $623 \pm 2$ \\
\hline True Density $(\mathrm{g} / \mathrm{cm} 3)$ & $1.523 \pm 0.005$ & $1.515 \pm 0.004$ & $1.522 \pm 0.007$ & $1.519 \pm 0.002$ & $1.535 \pm 0.004$ & $1.472 \pm 0.012$ & $1.458 \pm 0.009$ & $1.274 \pm 0.003$ \\
\hline Tapped density $(\mathrm{g} / \mathrm{cm} 3)$ & $0.906 \pm 0.010$ & $0.761 \pm 0.029$ & $0.455 \pm 0$ & $0.573 \pm 0.004$ & $0.481 \pm 0$ & $0.656 \pm 0.005$ & $0.665 \pm 0.019$ & $0.509 \pm 0.008$ \\
\hline
\end{tabular}

Firstly, the tablet weight variation (Ph.Eur) -as a reliable indicator of the influence of powder flow properties on the manufacturing processes- was used to evaluate the ability of multiple flow parameters to predict with the success of tablet manufacturing. For that, only directly compressed materials (no lubricant added) were analyzed at this stage. The physical properties of the different MCC grades used were as follows; Vivapur® 101 had the finest particle size, with a median (Dv50) of $63.2 \mu \mathrm{m}$ in PSD studies (see Table. 1). Particles smaller than $100 \mu \mathrm{m}$ are usually cohesive, and are susceptible to triboelectrification and flow problems (25), which explains its known poor flowing properties. Avicel® PH grade 102 had a larger particle size $(\mathrm{Dv} 50=102.6 \mu \mathrm{m})$, and it has been widely used in direct compression. Moreover, grade 102 has been considered at the boundary between an acceptable and a poor flowing excipient for tableting in high-speed machines (4). For this reason, it has been used as a reference material against which the "goodness" of the flow properties of formulations may be judged. Avicel@ PH grade 200 had the largest median particle size (Dv50=189.3 $\mu \mathrm{m}$ ) and good compactability (29). Due to its superior flow it has been used as excipient to correct flow deficiencies in direct compression (29). All MCC grades exhibited particles with

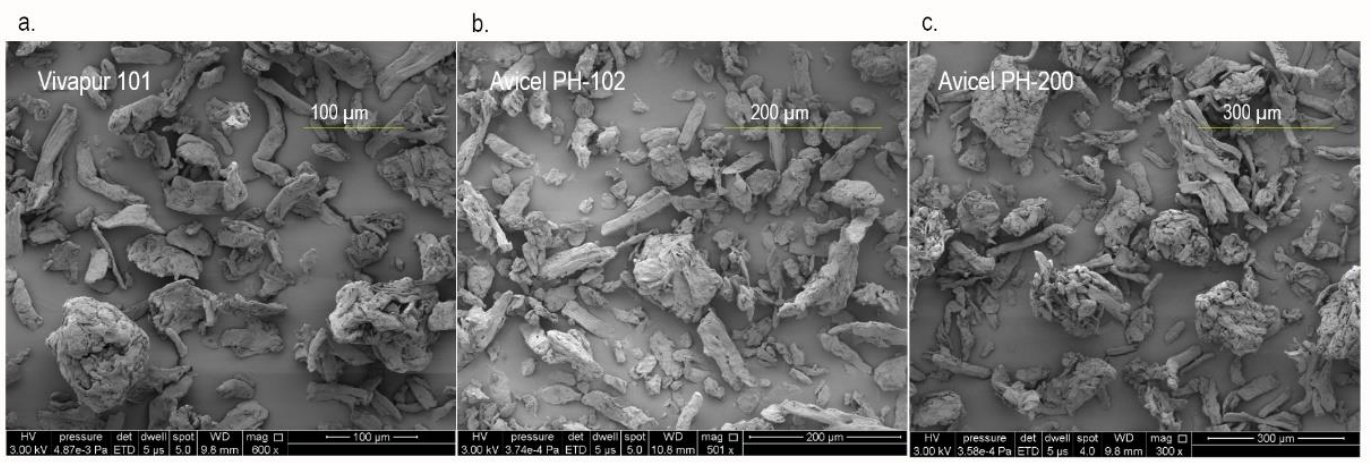

Fig. 3.

Scanning electron micrographs (SEMs) for microcrystalline cellulose grades: a. Vivapur@ 101, b. Avicel@ PH-102, and c. Avicel@ PH-200. 
In order to assess the filling capacity of MCC grades, the die-cavity volume was determined knowing the radius and the height. Subsequently, using the tapped densities of each material from tapping studies (see Table. 1), the theoretical mass for an optimum filling was calculated. This value was compared to the average obtained in the compression studies as shown in Fig. 4. Naturally, better powder flow properties resulted in increased die filling efficiency as shown in Fig.4. There was a clear difference between ideal weight (represented as a reference point in Fig.4), and experimental weight (represented as columns) for the different MCC grades. In addition, a progressive decrease in the standard deviation of tablet weight also reflected an increase in the filling efficiency of the die. Median particle size (Dv50) was positively correlated with tableting studies, confirming that a larger particle size, subjected to fewer intramolecular interactions, flows better into the die cavity. Consequently, the MCC grades can be ranked in increasing die filling efficiency order -a reflection of the powder flow properties- as their particle size increase (Vivapur® 101, Avicel@ PH-102, and Avicel@ PH-200).

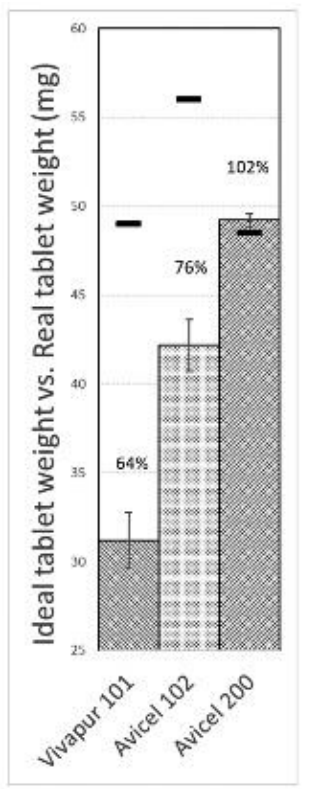

\section{Fig. 4.}

Die-cavity filling of MCC grades in tableting studies. Experimental weight represented as columns with standard deviation. Ideal-theoretical weight represented as a reference point for each material. Percentages represent the die filling efficiency (calculated from the experimental filling vs. the ideal filling).

The tablet weight variation (RSD) was used as reference method in the comparison as reported in Fig. 5a. Carr's index and flow through an orifice were in accordance with RSD values (Fig. 5c), being effective in assessing powder flowability. Still, flow through an orifice was unable to characterize the flow of MCC-grade 101 as the material was too cohesive. Compression data showed positive correlations with all powder rheometer dynamic properties. Even so, none of the powder rheometer parameters were able to classify and/or noticeably distinguish powder flow between the three grades of MCC, as the reported margin error of experimental data cannot be considered statistically significant (see Fig. 5b). As expected, static-flow parameters -FF and cohesion- were not correlated with tableting studies and have not been included as results. Therefore, powder rheometry was inefficient to predict the success of tablet manufacturing (based on the tablet weight RSD) of direct compression excipient MCC. Conversely, results suggested a positive correlation with the novel device presented here, as shown in Fig. 5d. MCC grades were ranked properly, with a significant statistical differentiation in flow characterization. The differences in processing time and sample amount required were also significantly different between the methods (Fig. 5). Additionally, as described in Ph.Eur (3,5), tests for minimum orifice diameter and high flow rate through a funnel-shape, as the novel method, most closely simulate high-velocity compression processes. In contrast, the small amount of material analyzed by the novel method cannot represent the actual compaction behaviour a powder may undergo during bulk transfer operations (e.g. powder packing during manufacturing processes or powder storage). This limitation may be considered in large-scale tablet manufacturing processes, where large amounts of powder behave differently from particle to bulk level. Nevertheless, none of the traditional tests gave a physical parameter directly linked to the flow at the press. 

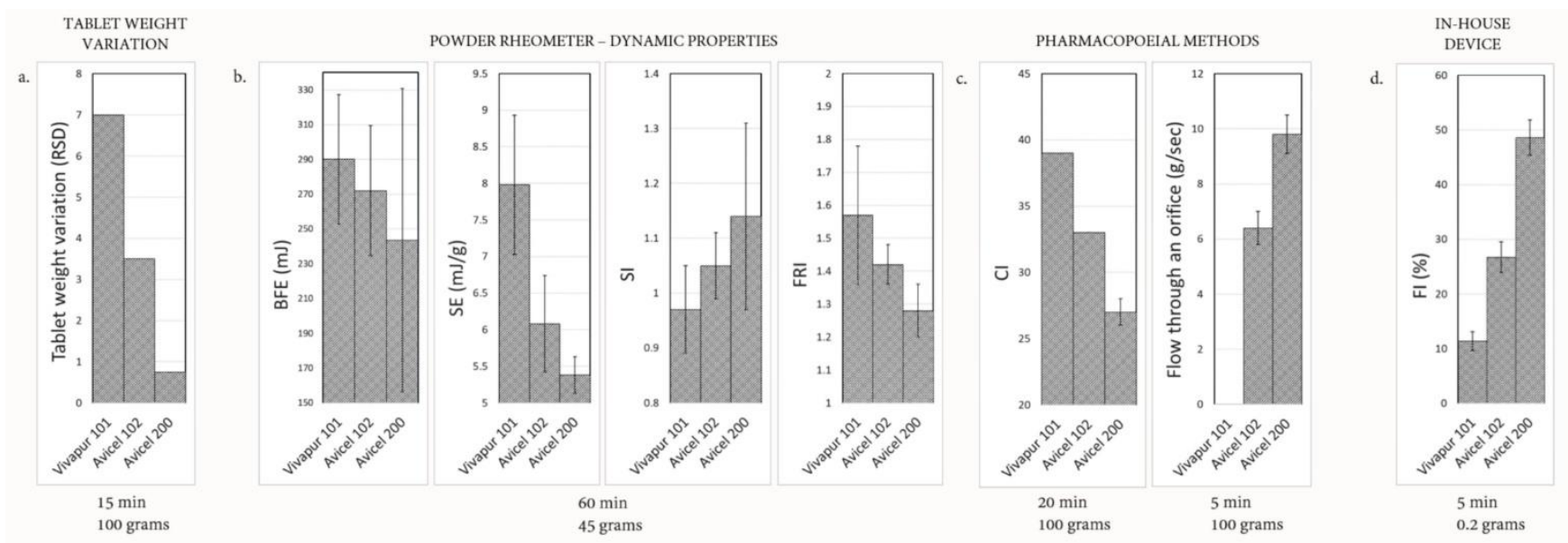

Fig. 5.

Powder flow characterization of MCC grades ( $\mathrm{n}=3$ ). The amount of material and time consumed for each MCC grade measurement is attached below each method. a. Tablet

weight variation (RSD). b. Powder rheometer. c. Pharmacopoeia methods. d. In-house device.

Secondly, to further investigate the relationship between flow characterization methods and the novel device, positive correlations with tableting studies -i.e. CI and flow through an orifice- were examined. All the studied materials were included in this analysis. The physical properties of sugar excipients (lactose $80 \mathrm{M}$ and 200M) exhibited particles with a relatively round and spherical shape as seen in Fig. $6 a-b$, and a Dv50 of $212.7 \mu \mathrm{m}$ and $40.1 \mu \mathrm{m}$ respectively (see Table 1 ). In the case of 200 Mesh sample, the wider size distribution and the smaller particle size contributes to a significant degree of interlocking and thus poor powder flow properties. Along with paracetamol, starch showed the lowest median particle size (Dv50=20.8 $\mu \mathrm{m})$, with highly spherical particles that formed agglomerates due to the predominant interparticular forces (Fig. 6c). API samples were composed of particles with sizes ranging from $41 \mu \mathrm{m}$ (Caffeine) to $20.8 \mu \mathrm{m}$ (Paracetamol) and of irregular shape, as illustrated in Fig. 7. This results in highly-cohesive behaviour for both materials. In the case of paracetamol, it exhibited a flattened shape, which is also easily affected by triboelectrification (34). Caffeine was made up of long, silky crystals conforming fleecy masses. Furthermore, in case of smaller particle size materials, the effect of moisture was easily observed during handling as aggregates formed, which means a deterioration of flow.

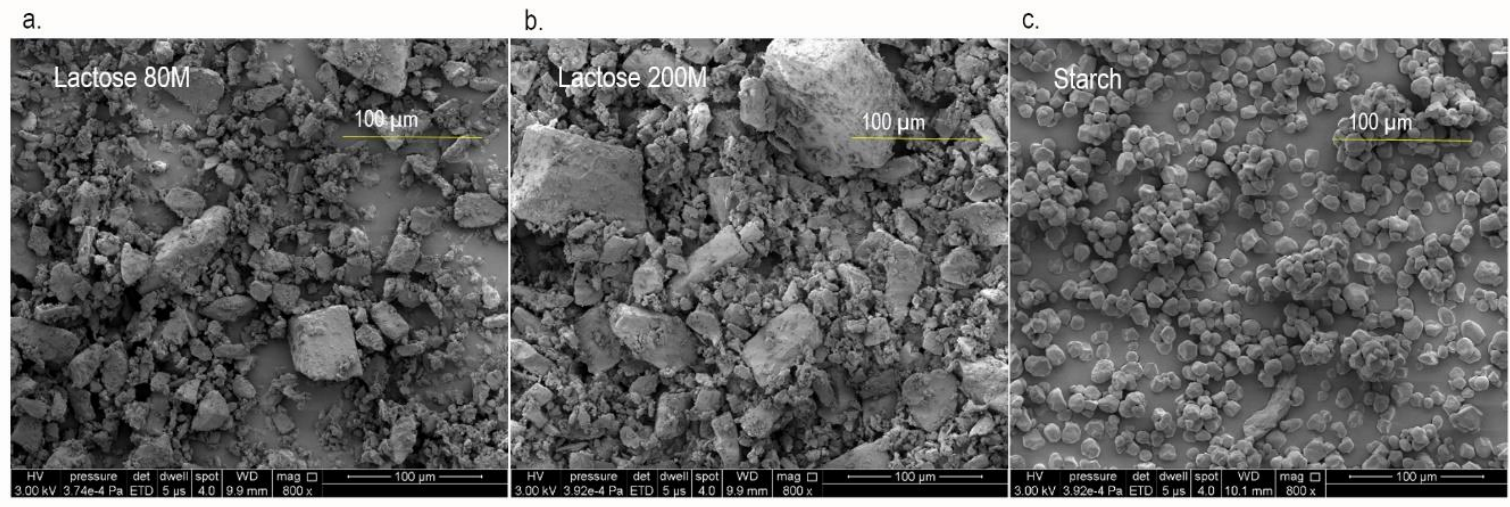

Fig. 6.

Scanning electron micrographs (SEMs) for sugars and starch: a. Lactose 80M, b. Lactose 200M, and c. Starch. 


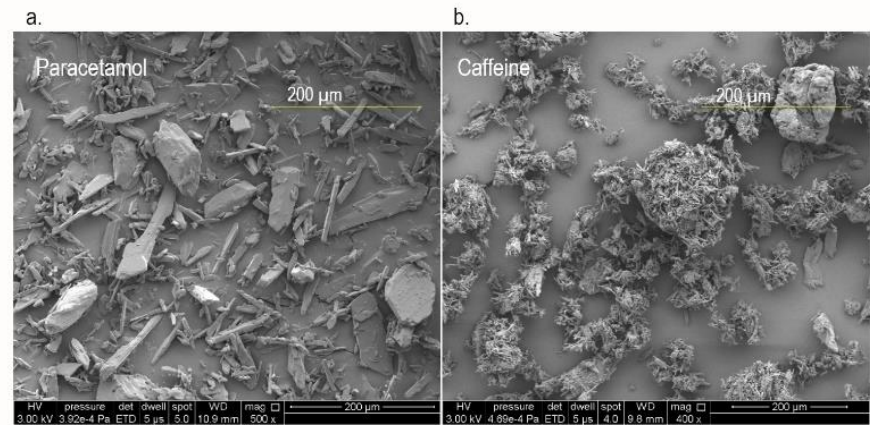

Fig. 7.

Scanning electron micrographs (SEMs) for API's: a. Paracetamol, and b. Caffeine.

Flow through an orifice has been proposed as one of the best methods to measure powder flowability in the pharmacopoeia (Ph.Eur), but the arching tendency of cohesive powders across the hopper opening impedes flow (15). Therefore, only free-flowing materials (Avicel ${ }^{\circledR}$ PH-102, Avicel® PH-200 and lactose $80 \mathrm{M}$ ) could be analyzed by this method. This validated the 102-grade MCC as the boundary between an acceptable and a poor flowing excipients, and classified lactose $80 \mathrm{M}$ as best-flowing material in this study (Flow rate; $12 \mathrm{~g} / \mathrm{s}$ for lactose $80 \mathrm{M}, 9.9 \mathrm{~g} / \mathrm{s}$ for Avicel PH-200 and $6.4 \mathrm{~g} / \mathrm{s}$ for Avicel PH-102). Moreover, in this method it is difficult to compare published results, as the flow is critically dependent on the specific procedure used to measure $(3,5)$.

The flow data obtained from the novel method were consistent with Carr's indices as shown in Fig. 8. MCC grades tested were ranked in the right order, depending on particle properties. Sugars were also properly classified, but the novel method was not sensitive enough to distinguish between goodflowing lactose $80 \mathrm{M}$ and Avicel ${ }^{\circledR} \mathrm{PH}-200$ as shown in the diagram in Fig. 8. Even so, despite not showing a significant statistical difference, the average

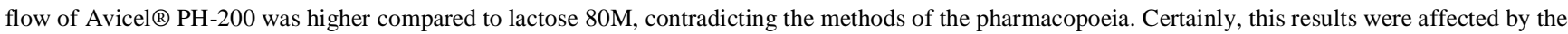
reasonably high humidity used in this study $(50 \% \mathrm{RH})$. Based on the optical evaluation of the flow in the novel device, we can expect better flow properties of Avicel® PH-200 compared to lactose 80M (see Fig. 8). A simple determination of Carr's indices of both substances at 20\%RH showed better flow properties on Avicel ${ }^{\text {PH }}$-200 (CI; 10 for Avicel PH-200 and 23 for lactose 80M) in contrast to those obtained at 50\%RH (CI; 27 for Avicel PH-200 and 22 for lactose $80 \mathrm{M}$ ). Considering these contradictory results and understanding that powder flowability is closely dependent to the test method used to measure, we can state that the new device successfully classified the flow of Avicel® PH-200 and lactose $80 \mathrm{M}$ in these specific conditions and small sample size. Nevertheless, future studies may evaluate subtle differences in flow caused by minor changes in RH conditions and the impact on tableting process. API's were in accordance with the CI and the novel method, revealing both slightly better flow properties on caffeine. However, CI was unable to properly characterize starch, indicating a similar flow to caffeine.

In contrast, the proposed novel method may not be the best for distinguishing between subtle differences of too cohesive samples, such as paracetamol and lactose 200M (Fig. 8). For this purpose shear-cell analysis considers the internal friction of the samples, providing a deeper insight on the flow behaviour of very cohesive materials. Future experiments to further develop the method will attempt to address the analysis of smaller particle sizes, approaching the nanoparticulate range (Dv50<100nm). The use of mixtures from cohesive materials and certain excipients as a glidant (e.g. porous nanosilica and magnesium stearate), as well as powder granules, will further evaluate the sensitivity of the method to small changes in powder flow caused by minor changes in sample characteristics. 

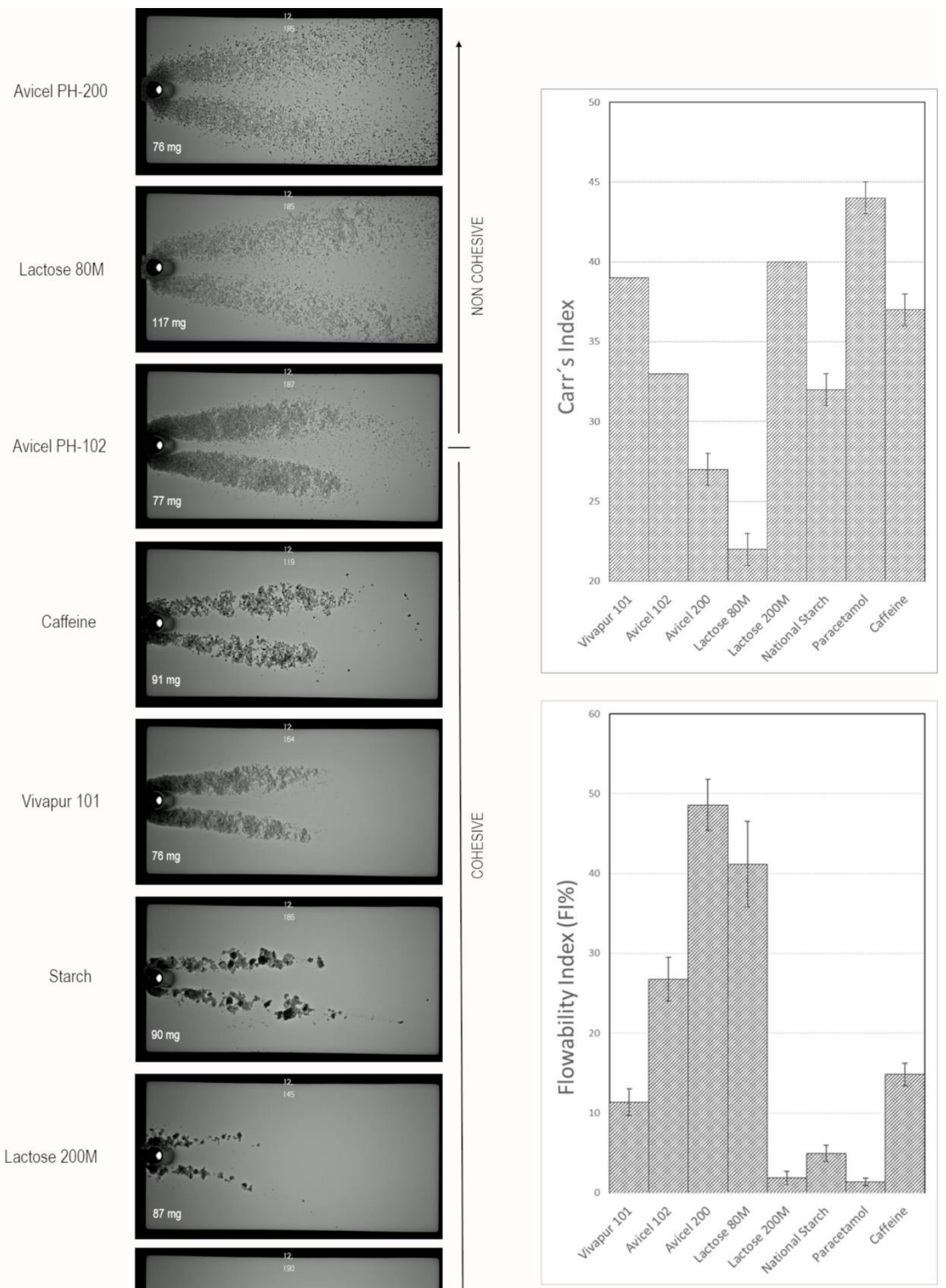

Lactose $200 \mathrm{M}$
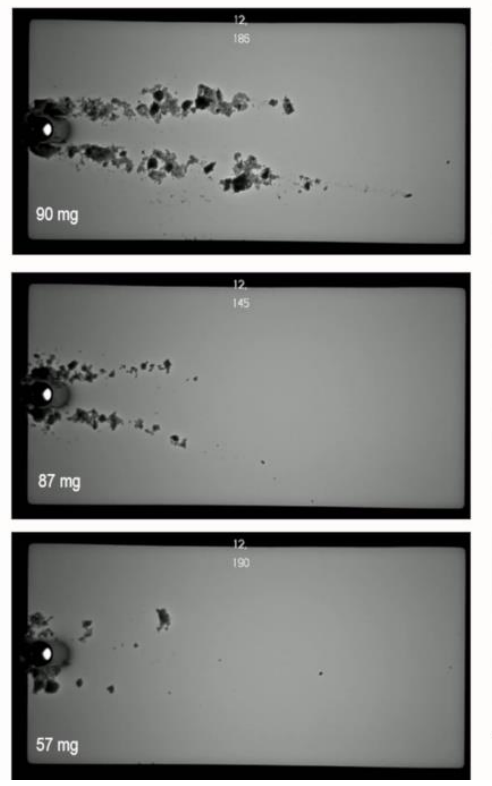

Paracetamol

\section{Fig. 8 .}

Novel In-house optical powder flow characterization. From the top to the bottom in descending order of flow. Carr's Index and In-house device flow profiles are attached. 
Powder flow in the introduced method was apparently subjected to a fluidization process. This involved airflow counter to the force of gravity through the bed of powder, where particles were readily separated from each other. Naturally, cohesive powders did not fluidize easily. Fluidized feed systems may be used in tablet manufacturing processes in which high feed rates or very fine powders are involved $(3,22)$. In the eccentric tablet machine used in this study, the powder feeder oscillates back and forth filling the die cavity. This may result in some kind of fluidization process, properly characterized by the novel device. Nonetheless, the application and the equipment must be considered, and it would be unwise to generalize these results to other equipment such as rotary tableting machines, where fluidization hardly occurs.

\section{CONCLUSIONS}

The present study introduces a promising method suitable for providing relevant information on powder flow properties, especially to reproduce the success of tablet manufacturing. The method is simple, sensitive, repeatable, and it is capable of classifying a wide range of pharmaceutical powders at a small, laboratory scale (sample size under $150 \mathrm{mg}$ ). These sample sizes together with the measurement method itself, reproduce similar conditions to the tableting process. In addition, the method is safe and sample-sparing, there is no contamination or environmental exposure and the material used in the

In modern industry, typically, optical evaluation tests are conducted manually with large amounts of substance and produce inconsistent results. In contrast, the automated ability for direct optical evaluation of powder flow in the novel device demonstrated to be a suitable test to provide unique information on powder flow properties. It is foreseen that the studied method could be important in drug research, especially in early preclinical research when the amount of new substance is limited and extremely expensive.

\section{ACKNOWLEDGEMENTS}

The authors gratefully acknowledge financial support from the Finnish Cultural Foundation -Suomen Kulttuurirahasto Foundation- [2018], and Finnish National Agency for Education (EDUFI) -Opetushallitus- [Grant Number TM-19-11041, 2019]. AM Healy acknowledges a research grant from Science Foundation Ireland (SFI) [Grant Number 12/RC/2275_P2], co-funded under the European Regional Development Fund.

\section{REFERENCES}

1. Tan, G., Morton, D., \& Larson, I., 2015. On the Methods to Measure Powder Flow. CPD. 21(40), 5751-5765.

2. Tay, J. Y. S., Liew, C. V., \& Heng, P. W. S., 2017. Powder Flow Testing: Judicious Choice of Test Methods. AAPS PharmSciTech. 18(5), 1843-1854.

3. J.J. Prescott, R.A. Barnum., 2000. On powder flowability. Pharm Technology. 24(10), 60-84.

355 4. Sun, C. C., 2010. Setting the bar for powder flow properties in successful high speed tableting. Powder Technology. 201(1), 106-108.

5. European Pharmacopoeia 10.0 ${ }^{\text {th }}$ Ed. (2.9.36. Powder Flow).

6. Freeman, R., 2007. Measuring the flow properties of consolidated, conditioned and aerated powders - A comparative study using a powder rheometer and a rotational shear cell. Powder Technology. 174(1-2), 25-33.

7. Schwedes, J., 2003. Review on testers for measuring flow properties of bulk solids. Granular Matter. 5(1), 1-43.

8. J. Duran., 2000. Sands, Powders and Grains. An Introduction to the Physics of Granular Materials. Paris, France, Springer.

9. Rowe, R.C., Sheskey, P.J. and Quinn, M.E., 2009 Handbook of Pharmaceutical Excipients. 6th Edition, Pharmaceutical Press. 
10. Taylor, M. K., Ginsburg, J., Hickey, A. J., \& Gheyas, F., 2000. Composite method to quantify powder flow as a screening method in early tablet or capsule formulation development. AAPS PharmSciTech. 1(3), 20-30.

11. Krantz, M., Zhang, H., \& Zhu, J., 2009. Characterization of powder flow: Static and dynamic testing. Powder Technology. 194(3), $239-245$.

12. Visser, J., 1989. Van der Waals and other cohesive forces affecting powder fluidization. Powder Technology. 58(1), 1-10.

13. Michael E. Aulton., 1988. Pharmaceutics. The science of dosage form design. Leicester, UK. Churchill Livingstone.

14. Hare, C., U. Zafar, M. Ghadiri, T. Freeman, J. Clayton, and M. J. Murtagh. 2015. Analysis of the dynamics of the FT4 powder rheometer. Powder Technol. 285:123-27.

370

15. Fitzpatrick, John \& Barringer, S.A \& Iqbal, Tariq., 2004. Flow Property Measurement of Food Powders and Sensitivity of Jenike's Hopper Design Methodology to the Measured Values. Journal of Food Engineering. 61, 399-405.

16. Rios, M., 2006. Developments in powder flow testing. Pharmaceutical Technology. 30, 38-49.

17. Souza, D. O. C., \& Menegalli, F. C. (2011). Image analysis: Statistical study of particle size distribution and shape characterization. Powder Technology, 214(1), 57-63.

375

18. Navaneethan, Charu \& Missaghi, Shahrzad \& Fassihi, Reza., 2005. Application of powder rheometer to determine powder flow properties and lubrication efficiency of pharmaceutical particulate systems. AAPS PharmSciTech. 6(3), 398-404.

19. Räsänen, E., Antikainen, O., \& Yliruusi, J., 2003. A new method to predict flowability using a microscale fluid bed. AAPS PharmSciTech. 4(4), 418424.

20. Hou, H., \& Sun, C. C., 2008. Quantifying Effects of Particulate Properties on Powder Flow Properties Using a Ring Shear Tester. Journal of

Pharmaceutical Sciences. 97(9), 4030-4039.

21. Crowder, Timothy \& Hickey, Anthony., 1999. An Instrument for Rapid Powder Flow Measurement and Temporal Fractal Analysis. Particle \&

Particle Systems Characterization. 16, 32-34.

22. Hans Mollet \& Arnold Grubenmann., 2001. Formulation Technology. Emulsions, Suspensions, Solid Forms. Mörlenbach, Germany. WILEY-VCH.

23. Jiang, Yanbin \& Matsusaka, Shuji \& Masuda, Hiroaki \& Qian, Yu., 2009. Development of Measurement System for Powder Flowability Based on

Vibrating Capillary Method. Powder Technology. 188, 242-247.

24. Seppälä, Kari \& Heinämäki, Jyrki \& Hatara, Juha \& Seppälä, Lassi \& Yliruusi, Jouko., 2010. Development of a New Method to Get a Reliable Powder Flow Characteristics Using Only 1 to $2 \mathrm{~g}$ of Powder. AAPS PharmSciTech. 11, 402-408.

25. J Staniforth., 2002. The science of dosage form design. $2^{\text {nd }}$ Edition. Powder flow, in Aulton ME Pharmaceutics.

26. Freeman, R., 2004. Predicting flowability and characterizing powders. Pharm Technol Eur. 16(1), 41-43.

27. Shi, Limin \& Chattoraj, Sayantan \& Sun, Changquan., 2011. Reproducibility of flow properties of microcrystalline cellulose - Avicel PH102. Powder Technology. 212, 253-257.

28. Freeman, R., \& Fu, X., 2008. Characterisation of powder bulk, dynamic flow and shear properties in relation to die filling. Powder Metallurgy. 51(3), 196-201.

29. Rojas J, Lopez A, Guisao S, Ortiz C., 2011 Evaluation of several microcrystalline celluloses obtained from agricultural by-products. J Adv Pharm

30. Jivraj, Mira \& Martini, Luigi \& Thomson, Carol., 2000. An overview of the different excipients useful for the direct compression of tablets.

Pharmaceutical Science \& Technology Today. 3, 58-63.

31. Carson, J.W., 1975. Applying bulk solid flow principles to metal powders. Int. J. Powder Metall. 11(4), 287-301. 
32. Jenike, Andrew W., 1970. Storage and flow of solids (Rev. ed). University of Utah, Salt Lake City, Utah.

33. Chuan-Yu Wu, Brian Armstrong \& Nikolaus Vlachos., 2012. Characterization of Powder Flowability for Die Filling, Particulate Science and Technology, 30(4), 378-389.

34. Naik, Shivangi \& Mukherjee, Raj \& Chaudhuri, Bodhisattwa., 2016. Triboelectrification: A review of experimental and mechanistic modeling approaches with a special focus on pharmaceutical powders. International Journal of Pharmaceutics. 510(1), 375-85.

35. Mühlich, P., Case, W., Hörmansperger, J., Beyrer, M., \& Windhab, E. J. 2016. Particle Image Velocimetry (PIV) in food powders during Vacuum 405 Steam-Vacuum (VSV) treatment. Powder Technology, 299, 1-8.

36. Mahmoudi, S., Elserfy, K., Cheng, S., Chan, H. K., Hebbink, G., \& Kourmatzis, A. 2019. Fluidisation characteristics of lactose powders in simple turbulent channel flows. Experimental Thermal and Fluid Science, 103(October 2018), 201-213.

37. Hagemeier, T., Börner, M., Bück, A., \& Tsotsas, E. 2015. A comparative study on optical techniques for the estimation of granular flow velocities. Chemical Engineering Science, 131, 63-75.

410 38. Higham, Jonathan \& Brevis, W.. (2019). When, what and how image transformation techniques should be used to reduce error in Particle Image Velocimetry data?. Flow Measurement and Instrumentation. 66:79-85, 2019.

39. Kou, X., Wereley, S. T., Heng, P. W. S., Chan, L. W., \& Carvajal, M. T. (2016). Powder dispersion mechanisms within a dry powder inhaler using microscale particle image velocimetry. International Journal of Pharmaceutics, 514(2), 445-455.

40. Pasquali, I., Merusi, C., Brambilla, G., Long, E. J., Hargrave, G. K., \& Versteeg, H. K. (2015). Optical diagnostics study of air flow and powder

415 fluidisation in Nexthaler @ — Part I: Studies with lactose placebo formulation. International Journal of Pharmaceutics, 496(2), $780-791$.

41. Kou, X., Heng, P. W. S., Chan, L. W., Wereley, S. T., \& Carvajal, M. T. (2019). Effect of Roughness on the Dispersion of Dry Powders for Inhalation: a Dynamic Visualization Perspective. AAPS PharmSciTech, 20(7).

42. Elserfy, K., Kourmatzis, A., Chan, H.-K., Walenga, R., \& Cheng, S. (2020). Effect of an upstream grid on the fluidization of pharmaceutical carrier powders. International Journal of Pharmaceutics, 119079. doi:10.1016/j.ijpharm.2020.119079 